\title{
Modelo computacional para suporte à decisão em áreas irrigadas. Parte II: Testes e aplicação
}

\author{
Paulo A. Ferreira ${ }^{1}$, João C. F. Borges Júnior ${ }^{2}$, Bettina Hedden-Dunkhorst ${ }^{3}$ \& Camilo de L. T. de Andrade ${ }^{4}$
}

\begin{abstract}
RESUMO
Apresentou-se, na Parte I desta pesquisa, o desenvolvimento de um modelo computacional denominado MCID, para suporte à tomada de decisão quanto ao planejamento e manejo de projetos de irrigação e/ou drenagem. Objetivou-se, na Parte II, testar e aplicar o MCID. No teste comparativo com o programa DRAINMOD, espaçamentos entre drenos, obtidos com o MCID, foram ligeiramente maiores ou idênticos. Os espaçamentos advindos com o MCID e o DRAINMOD foram consideravelmente maiores que os obtidos por meio de metodologias tradicionais de dimensionamento de sistemas de drenagem. A produtividade relativa total, YRT, obtida com o MCID foi, em geral, inferior à conseguida com o DRAINMOD, devido a diferenças de metodologia ao se estimar a produtividade da cultura em resposta ao déficit hídrico. Na comparação com o programa CROPWAT, obtiveram-se resultados muito próximos para (YRT) e evapotranspiração real. O modelo desenvolvido foi aplicado para as condições do Projeto Jaíba, MG, para culturas perenes e anuais cultivadas em diferentes épocas. Os resultados dos testes e aplicações indicaram a potencialidade do MCID como ferramenta de apoio à decisão em projetos de irrigação e/ou drenagem.
\end{abstract}

Palavras-chave: manejo solo-água-planta-salinidade, produtividade relativa, análise financeira

\section{Computer model for decision support in irrigated areas. Part II: Tests and application}

\begin{abstract}
Part I of this research presented the development of a decision support model, called MCID, for planning and managing irrigation and/or drainage projects. Part II is aimed at testing and applying MCID. In a comparative test with the DRAINMOD model, drain spacings obtained with MCID were slightly larger or identical. The spacings obtained with MCID and DRAINMOD were considerably larger than those obtained through traditional methodologies of design of drainage systems. The relative crop yield (YRT) obtained with MCID was, in general, lower than the one obtained with DRAINMOD due to differences in the estimate of crop response to water deficit. In comparison with CROPWAT, very close results for YRT and for actual evapotranspiration were obtained. The developed model was applied to perennial and annual crops during different cropping periods grown at the Jaiba Irrigation Project, Brazil. The results of tests and applications show the potential of MCID as a decision support tool for irrigation and/or drainage projects.
\end{abstract}

Key words: soil-water-plant-salinity management, relative yield, financial analysis 


\section{INTRODUÇÃO}

Na Parte I deste trabalho apresentou-se o desenvolvimento e análise de sensibilidade de um modelo computacional denominado MCID, para suporte à tomada de decisão em projetos de irrigação e/ou drenagem.

O MCID é aplicável em nível de unidade de produção. Unidade de produção, aqui, refere-se a uma área sujeita a sistemas e manejo de irrigação e/ou drenagem específicos, com determinada cultura ou seqüência de culturas cultivadas em um ano, em um mesmo tipo de solo no que se refere às características físicas que afetam o movimento de água. O balanço hídrico e de sais na zona radicular, bem como as estimativas da profundidade do lençol freático e da vazão nos drenos, são conduzidos em base diária. A entrada de dados e o acesso aos resultados são feitos por meio da interface gráfica do programa.

A aplicação deste modelo visa obter informações relativas ao efeito de diferentes práticas de manejo de irrigação e configurações do sistema de drenagem sobre a produtividade e o retorno financeiro. Estas informações podem ser empregadas em estudos de otimização de padrão de cultivo em relação ao retorno financeiro e ao uso da água, associados a análise de risco com base em simulações (Borges Júnior et al., 2003).

Objetivou-se, com este trabalho, testar e aplicar o modelo MCID para diferentes bases de dados de entrada, visando verificar sua potencialidade como ferramenta de apoio à tomada de decisão quanto ao manejo e planejamento de projetos de irrigação e/ou drenagem.

\section{MATERIAL E MÉTODOS}

Realizaram-se estimativas de produtividade relativa total e testes comparativos do modelo MCID com o programa DRAINMOD e com metodologias tradicionais de dimensionamento de sistemas de drenagem (Esquema 1) além de testes comparativos do modelo MCID com o programa CROPWAT da FAO, quanto à previsão da produtividade relativa da cultura, evapotranspiração real e depleção de água na zona radicular (Esquema 2). As descrições dos esquemas de modelagem 1 e 2 foram dadas na Parte I deste trabalho. O desempenho do modelo MCID foi, então, estudado, utilizandose dados do Projeto Jaíba, MG.

\section{Desempenho do modelo MCID - Esquema 1}

Os procedimentos implementados no modelo para determinar a variação do lençol freático são, basicamente, aqueles do modelo PRODREN (Borges Júnior et al., 2001; Ferreira et al., 2001), com pequenas diferenças em relação à implementação da equação de drenagem de Kraijenhoff van de Leur Maasland. As diferenças metodológicas mais expressivas foram aquelas relacionadas com a estimativa da produtividade relativa em resposta ao déficit hídrico, na zona radicular. No MCID, foi implementada a metodologia prescrita pela FAO (Allen et al., 1998) enquanto no PRODREN a metodologia é similar à utilizada no DRAINMOD (Skaggs, 1981; Skaggs, 1999).
Os dimensionamentos de sistemas de drenagem, obtidos com o MCID, foram comparados com os obtidos com o modelo DRAINMOD e com as metodologias tradicionalmente empregadas no Brasil, que utilizam critérios preconizados por Pizarro (1985) e Cruciani (1987).

Estimativas das produtividades relativas totais obtidas com o MCID e com o DRAINMOD também foram comparadas; não se considerou o estresse devido à salinidade.

A base de dados utilizada foi apresentada por Duarte (1997), exceto alguns dados de cultura requeridos apenas para o MCID. A base de dados de entrada constituiu-se de:

\section{Clima}

Utilizaram-se séries históricas de dados diários de precipitação e evapotranspiração potencial da região de Piracicaba, para o período de 1974 a 1994, num total de 21 anos.

\section{Solo}

Realizaram-se simulações empregando-se três tipos de solo, com a seguinte classificação textural: solo 1 - francoargiloso; solo 2 - argiloso, e solo 3 - franco argilo-siltoso.

Para esses três tipos de solo, os teores volumétricos de água na saturação $\left(\theta_{\mathrm{S}}\right)$, capacidade de campo $\left(\theta_{\mathrm{CC}}\right)$, ponto de murcha permanente $\left(\theta_{\mathrm{PM}}\right)$ e em equilíbrio com uma tensão de $6 \mathrm{kPa}\left(\theta_{\mathrm{Pd}}\right)$, utilizado no cálculo da porosidade drenável, como também as condutividades hidráulicas horizontal (Kho) e vertical (Kvo) em meio saturado, são apresentados na Tabela 1.

Tabela 1. Teores volumétricos de água $\left(\mathrm{m}^{3} \mathrm{~m}^{-3}\right)$ na saturação $(\theta s)$, capacidade de campo $\left(\theta_{C C}\right)$, ponto de murcha $\left(\theta_{\mathrm{PM}}\right)$ e em equilíbrio com a tensão de $6 \mathrm{kPa}\left(\theta_{P_{d}}\right)$ e condutividades hidráulicas horizontal (Kho) e vertical (Kvo) em meio saturado

\begin{tabular}{|c|c|c|c|c|c|c|}
\hline \multirow[t]{2}{*}{ Solo } & $\theta s$ & $\theta_{\text {cc }}$ & $\theta_{\mathrm{PM}}$ & $\theta_{\text {Pd }}$ & Kho & Kvo \\
\hline & \multicolumn{4}{|c|}{$\mathrm{m}^{3} \mathrm{~m}^{-3}$} & \multicolumn{2}{|c|}{$\left(m\right.$ dia $\left.^{-1}\right)$} \\
\hline Franco-argiloso & 0,572 & 0,372 & 0,189 & 0,434 & 1,00 & 0,50 \\
\hline Argila & 0,645 & 0,546 & 0,444 & 0,577 & 0,50 & 0,25 \\
\hline $\begin{array}{l}\text { Franco-argilo- } \\
\text { siltoso }\end{array}$ & 0,494 & 0,427 & 0,329 & 0,443 & 0,10 & 0,05 \\
\hline
\end{tabular}

Os parâmetros de entrada do modelo de van GenuchtenMulem (van Genuchten, 1980) são apresentados na Tabela 2.

A profundidade da camada impermeável, Dci, considerada nos cálculos, foi $5 \mathrm{~m}$. O teor de água inicial do solo, $\theta_{0}$, foi aquele em equilíbrio com a tensão de $6 \mathrm{kPa}$. A altura inicial do lençol freático em relação ao plano de assentamento dos drenos, $\mathrm{hlf}_{0}$, foi considerada de $0,6 \mathrm{~m}$. Assumiu-se que os parâmetros $\theta_{0}$ e hlf $f_{0}$ têm influência inexpressiva sobre os resultados, em virtude da extensão do período em que a simulação foi

Tabela 2. Parâmetros do modelo de van Genuchten-Mualem

\begin{tabular}{|c|c|c|c|c|c|}
\hline \multirow[t]{2}{*}{ Solo } & $\theta_{\mathrm{S}}$ & $\theta r$ & \multirow{2}{*}{ m } & \multirow{2}{*}{ N } & \multirow{2}{*}{$\begin{array}{c}\alpha \\
\left(\mathrm{cm}^{-1}\right)\end{array}$} \\
\hline & \multicolumn{2}{|c|}{$\left(m^{3} m^{-3}\right)$} & & & \\
\hline Franco-argiloso & 0,572 & 0,112 & 0,2117 & 1,2685 & 0,0538 \\
\hline Argila & 0,645 & 0,363 & 0,1532 & 1,1809 & 0,0654 \\
\hline $\begin{array}{l}\text { Franco-argilo- } \\
\text { siltoso }\end{array}$ & 0,494 & 0,073 & 0,0633 & 1,0676 & 0,1010 \\
\hline
\end{tabular}


feita.

\section{Cultura}

A cultura sob simulação foi o milho, plantada no dia 8 de outubro e colhida no dia 14 de fevereiro, totalizando o ciclo de 130 dias. Os dados de variação da profundidade efetiva do sistema radicular e os coeficientes de estresse da cultura, quanto ao excesso de água no solo, foram os mesmos utilizados por Duarte (1997).

Os parâmetros fator de resposta da cultura, $\mathrm{K}_{\mathrm{y}}$, fator de disponibilidade, $\mathrm{f}$, e duração dos diferentes estádios foram empregados como dados de entrada para o MCID. O valor de $K_{y}$ foi aquele relatado por Doorenbos \& Kassam (1979). O fator de disponibilidade e a duração dos diferentes estádios foram obtidos da base de dados do programa CROPWAT. O estádio IV foi diminuído em 5 dias, para ajustar-se ao ciclo de 130 dias, típico da cultura plantada naquela data. Os parâmetros $K_{y}$, f e a duração dos estádios são apresentados na Tabela 3. Os parâmetros relacionados ao estresse da cultura em

Tabela 3. Valores do fator de resposta da cultura, $K_{y}$ (decimal), fator de disponibilidade, $f$ (decimal) e duração dos estádios de desenvolvimento I, II, III e IV e de todo o ciclo, para a cultura do milho (dias)

\begin{tabular}{cccccc}
\hline Fator & I & II & III & IV & Todo o ciclo \\
$\mathrm{K}_{\mathrm{y}}$ & 0,4 & 0,4 & 1,3 & 0,5 & 1,25 \\
$\mathrm{f}$ & 0,50 & 0,50 & 0,50 & 0,80 & - \\
\hline Duração & 25 & 40 & 40 & 25 & 130 \\
\hline
\end{tabular}

resposta ao déficit hídrico, empregados no DRAINMOD, foram os mesmos utilizados por Duarte (1997).

\section{Sistemas de drenagem}

As análises foram efetuadas para sistemas de drenagem construídos com drenos laterais cobertos, com espaçamentos de 5 a $100 \mathrm{~m}$, em incrementos de $5 \mathrm{~m}$ (20 espaçamentos), profundidade de $1,2 \mathrm{~m}$ e diâmetro efetivo de drenos de $0,1 \mathrm{~m}$.

\section{Dados econômicos}

Os parâmetros de entrada utilizados no cálculo do valor presente líquido por unidade de área, VPL $\left(\mathrm{R} \$ \mathrm{ha}^{-1}\right)$, são apresentados na Tabela 4.

\section{Outros dados}

Nas simulações, considerou-se que toda lâmina de chuva infiltra, o que é tido como critério de segurança no dimensionamento de sistemas de drenagem; não foram considerados o escoamento superficial nem a ocorrência de sípege (seepage).

\section{Desempenho do modelo MCID - Esquema 2}

Realizaram-se testes objetivando-se comparar o desempenho do modelo quanto a previsão da produtividade relativa total (YRT), evapotranspiração real (ETr, mm) e depleção de água na zona radicular (Dr, mm). Os resultados do MCID foram comparados com os obtidos com o programa CROPWAT
Tabela 4. Parâmetros de entrada utilizados no cálculo do valor presente líquido

\begin{tabular}{|c|c|}
\hline Parâmetro & Valor \\
\hline Vida econômica do projeto & 25 anos \\
\hline Taxa de desconto & $12 \%$ ao ano \\
\hline Produtividade potencial (Yp) & 12 tha $^{-1}$ \\
\hline Preço de venda $(P)$ & $\mathrm{R} \$ 108,33 \mathrm{t}^{-1}$ \\
\hline Custo de produção da cultura & $\mathrm{R} \$ 600,00 \mathrm{ha}^{-1}$ \\
\hline Área de plantio & 1 ha \\
\hline $\begin{array}{l}\text { Custo de saneamento e abertura dos } \\
\text { coletores (CSAC) }\end{array}$ & $\mathrm{R} \$ 350,00 \mathrm{ha}^{-1}$ \\
\hline $\begin{array}{l}\text { Custo do metro de dreno subterrâneo } \\
\text { instalado }\end{array}$ & $\mathrm{R} \$ 5,00 \mathrm{~m}^{-1}$ \\
\hline Custo de manutenção dos coletores & $\begin{array}{c}\mathrm{R} \$ 10,50{\text { (ha ano })^{-1}} \\
(3 \% \text { do valor de CSAC) }\end{array}$ \\
\hline $\begin{array}{l}\text { Custo de manutenção da rede subterrânea } \\
\text { de drenos }\end{array}$ & $\begin{array}{l}0,5 \% \text { do custo de } \\
\text { instalação por ano }\end{array}$ \\
\hline
\end{tabular}

(CROPWAT 4 Windows version 4.3 - Land and Water Development Division - FAO). Não foram considerados decréscimos de produtividade devido ao excesso de água no solo e à salinidade, já que o CROPWAT opera apenas com estresse devido ao déficit hídrico; assim, a produtividade relativa total é igual à produtividade relativa em resposta ao déficit hídrico, YRD. A base de dados de entrada é descrita a seguir.

\section{Dados climáticos}

Utilizaram-se dados da base CLIMWAT, que opera junto ao CROPWAT, para a cidade de Januária, MG. Esses são dados mensais médios de precipitação e evapotranspiração de referência, $\mathrm{ET}_{0}$. Dados médios podem ser utilizados no MCID, na ausência de séries históricas, embora neste caso não seja possível a execução dos procedimentos estatísticos implementados no programa.

No CLIMWAT, os dados de precipitação são fornecidos em mm mês ${ }^{-1}$ e os dados de $\mathrm{ET}_{0}$ em $\mathrm{mm} \mathrm{d}^{-1}$. Os procedimentos para distribuição dos dados mensais em base diária foram escolhidos de modo a se ajustarem às metodologias disponíveis nos dois modelos. Na distribuição da precipitação, foram considerados seis dias de chuva por mês, o que equivale a uma agregação de chuvas a cada cinco dias, que é o dado de entrada no CROPWAT; nele, ao contrário do que ocorre para o MCID, não é possível considerar diferentes números de dias de chuva por mês, em diferentes meses. Admitiu-se que toda a chuva infiltrou, dado ao baixo índice pluviométrico durante o período de cultivo. $\mathrm{A} \mathrm{ET}_{0}$ foi distribuída uniformemente para os dias dos meses (outras metodologias são disponíveis no CROPWAT).

\section{Dados de solo}

Foi considerado um solo de textura média, o qual consta na base de dados do CROPWAT, com a disponibilidade total de água no solo, DTA, igual a $1,4 \mathrm{~mm} \mathrm{~cm}^{-1}$. 


\section{Cultura}

A cultura considerada nos testes foi o feijão, plantada no dia $1^{\circ}$ de maio, cujos parâmetros foram tomados da base de dados que acompanha o CROPWAT, no arquivo BEANSDR.CRO. As estimativas de evapotranspiração real foram feitas empregando-se o coeficiente de cultura, $\mathrm{K}_{\mathrm{c}}$ (no CROPWAT, não é possível trabalhar com o coeficiente basal de cultura, $\mathrm{K}_{\mathrm{cb}}$ ). Considerou-se fator de resposta da cultura, $\mathrm{K}_{\mathrm{y}}$, para todo o ciclo, visto que o CROPWAT não processa dados de $\mathrm{K}_{\mathrm{y}}$ para os estádios. Quanto ao fator de disponibilidade, f, foi necessário utilizar-se um valor constante devido a diferenças do processamento desse fator nos dois modelos. No MCID, f é constante em cada estádio, enquanto no CROPWAT é considerado variável para os estádios I, II e IV, ocorrendo

Tabela 5. Valores do fator de resposta da cultura, $K_{y}$ (decimal), fator de disponibilidade, $f$ (decimal), e duração dos estádios de desenvolvimento I, II, III e IV e para todo o ciclo, para a cultura do feijão (dias)

\begin{tabular}{cccccc}
\hline Fator & I & II & III & IV & Todo o ciclo \\
$\mathrm{K}_{\mathrm{y}}$ & - & - & - & - & 1,15 \\
$\mathrm{f}$ & - & - & - & - & 0,45 \\
$\mathrm{~K}_{\mathrm{c}}$ & 0,4 & - & 1,15 & ${ }^{*} 0,35$ & - \\
Duração & 20 & 30 & 40 & 20 & 110 \\
\hline $\mathrm{K}_{\mathrm{c}}$ final & & & & &
\end{tabular}

interpolação para cada dia, nesses intervalos, com base nos valores de entrada de $\mathrm{f}$, para cada intervalo. Na Tabela 5 temse os dados de entrada para cultura.

\section{Critério de irrigação}

Consideraram-se irrigações com intervalos de 14 dias e lâmina constante de $20 \mathrm{~mm}$, iniciando-se no primeiro dia do ciclo. Como no CROPWAT os cálculos são iniciados a partir do primeiro dia do ciclo, com o teor de água inicial igual ao da capacidade de campo (é possível fazer correções nos valores do teor de água calculados com o programa ao longo do ciclo), foi considerada, para o MCID, uma irrigação de 20 $\mathrm{mm}$ no dia anterior ao dia de plantio, para elevar o teor de água do solo à capacidade de campo.

\section{Aplicação do MCID ao Projeto Jaíba}

O objetivo desta aplicação foi verificar a capacidade do modelo computacional quanto à construção de banco de dados sobre requerimento de irrigação e produtividade para várias culturas e diferentes épocas de cultivo. A base de dados empregada na aplicação é descrita a seguir.

\section{Dados climáticos}

Os seguintes dados climáticos mensais foram obtidos junto ao Distrito de Irrigação do Jaíba - DIJ: precipitação, médias das temperaturas máximas, médias das temperaturas mínimas, temperaturas médias, umidade relativa média do ar, insolação e velocidade do vento.

Exceto para a velocidade do vento, foram utilizados dados dos anos de 1991 a 2001. Para velocidade do vento, por não se dispor de dados para o período, utilizaram-se valores médios mensais considerando-se, conforme sugerido por Allen et al. (1998), um valor mínimo de $0,5 \mathrm{~m} \mathrm{~s}^{-1}$. A evapotranspiração de referência foi calculada por meio do programa REF-ET (Window Version 2.0), pelo método FAO Penman-Monteith.

Dados pluviométricos diários não foram empregados diretamente, por não estarem disponíveis, sem custos, para o período considerado. Com base na série histórica de dados pluviométricos diários de Mocambinho, distrito localizado no Projeto Jaíba, no período disponível (1976 a 1992), obtiveramse valores médios de número de dias de chuvas por mês, para os meses de janeiro a dezembro, iguais a 10, 9, 4, 4, 2, 1, 1, 1, $2,5,5$ e 8 , respectivamente.

\section{Dados de solo}

Valores médios de $\theta_{\mathrm{CC}}$ e $\theta_{\mathrm{PM}}$ foram obtidos de Quaresma Filho (2000), sendo $\theta_{\mathrm{CC}}$ igual a 0,260 e $\theta_{\mathrm{PM}}$ igual a 0,123 .

\section{Cultura}

As culturas consideradas na modelagem foram escolhidas com base na área plantada, valor da produção e/ou rentabilidade alcançada por essas culturas, nos anos de 2001 e 2002, conforme informado pelo Distrito de Irrigação do Projeto Jaíba (DIJ) e pela CODEVASF, $1^{a}$ Superintendência, para as categorias fruticultura, grãos e hortaliças. As culturas e épocas de cultivo (culturas anuais) consideradas foram:

- Culturas perenes: banana prata-anã, limão, mamão papaya e maracujá.

- Culturas anuais: abóbora japonesa (um cultivo por ano fevereiro a maio), algodão (dois cultivos por ano - março a junho e junho a setembro), cebola inverno (um cultivo por ano - abril a julho), cebola verão (um cultivo por ano - novembro a fevereiro), feijão (três cultivos por anos - janeiro a março, maio a julho, outubro a dezembro), melancia (um cultivo por ano - setembro a novembro), melão (dois cultivos por ano - março a maio e agosto a outubro), milho (dois cultivos por ano - dezembro a março e maio a agosto) e pepino para conserva (um cultivo por ano - abril a junho).

Considerou-se que as culturas eram plantadas no primeiro dia do mês inicial de cultivo, embora se possa considerar o plantio em outros dias no mês.

Valores de parâmetros das culturas são listados em Borges Júnior (2004), sendo suprimidos aqui por questão de espaço. Para a cultura da banana, trabalhou-se com o coeficiente basal de cultura $\left(\mathrm{K}_{\mathrm{cb}}\right)$, enquanto para as demais se trabalhou com o coeficiente único da cultura $\left(\mathrm{K}_{\mathrm{c}}\right)$. As produtividades potenciais das culturas (Yp) foram obtidas de informações do DIJ ou na literatura especializada.

\section{Irrigação}

O critério de irrigação, relativo ao momento de irrigar, foi diferenciado para as diversas culturas. Consideraram-se os sistemas de irrigação por aspersão, microaspersão e gotejamento. Dados relativos à irrigação são apresentados na Tabela 6. Quanto à lâmina de irrigação, considerou-se como critério irrigar para preencher $100 \%$ da capacidade real de água 
Tabela 6. Dados de entrada relativos à irrigação na aplicação para o Projeto Jaíba: fração do solo molhada pela irrigação (fwi, decimal), eficiência de distribuição de projeto (EDad, decimal), eficiência potencial de aplicação (EPa, decimal) e turno de rega

\begin{tabular}{|c|c|c|c|c|c|}
\hline Cultura & Sistema de irrigação & fwi & EDad & $\mathrm{EPa}$ & Turno de rega \\
\hline Abóbora japonesa & Aspersão convencional & 1 & 0,8 & 0,9 & Irrigar quando $\mathrm{Dr}=100 \%$ da $\mathrm{CRA}^{*}$ \\
\hline Algodão & Aspersão convencional & 1 & 0,8 & 0,9 & 7 dias (cessar 10 dias antes da colheita) \\
\hline Banana & Aspersão convencional & 1 & 0,8 & 0,9 & 7 dias \\
\hline Cebola inverno & Aspersão convencional & 1 & 0,8 & 0,9 & $\begin{array}{l}\text { Irrigar quando } \mathrm{Dr}=100 \% \text { da CRA } \\
\text { (cessar } 10 \text { dias antes da colheita) }\end{array}$ \\
\hline Cebola verão & Aspersão convencional & 1 & 0,8 & 0,9 & $\begin{array}{l}\text { Irrigar quando } \mathrm{Dr}=100 \% \text { da CRA } \\
\text { (cessar } 10 \text { dias antes da colheita) }\end{array}$ \\
\hline Feijão & Aspersão convencional & 1 & 0,8 & 0,9 & 7 dias (cessar 7 dias antes da colheita) \\
\hline Limão & Microaspersão & 0,7 & 0,8 & 1 & 1 dia \\
\hline Mamão & Microaspersão & 0,8 & 0,8 & 1 & 2 dias \\
\hline Maracujá & Gotejamento & 0,7 & 0,8 & 1 & 1 dia \\
\hline Melancia & Gotejamento & 0,8 & 0,8 & 1 & 1 dia \\
\hline Melão & Gotejamento & 0,8 & 0,8 & 1 & 1 dia \\
\hline Milho & Aspersão convencional & 1 & 0,8 & 0,9 & 7 dias (cessar 7 dias antes da colheita) \\
\hline Pepino & Aspersão convencional & 1 & 0,8 & 0,9 & 3 dias \\
\hline
\end{tabular}

* CRA - Capacidade real de água no solo (mm)

no solo, CRA (mm).

\section{Dados econômicos}

Dados concernentes aos preços dos produtos e aos custos de irrigação (taxas de água, custo da energia consumida na irrigação e consumo específico de energia) foram informados pelo DIJ para o ano de 2004, apresentados a seguir.

Taxas relativas a irrigação:

- K1 (tarifa relativa ao investimento público no perímetro

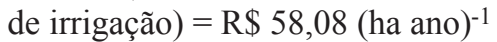

- K2 (tarifa referente aos gastos com operação e manutenção do sistema de irrigação do perímetro $)=\mathrm{R} \$ 17,82(1000$ $\left.\mathrm{m}^{3}\right)^{-1}$

Energia consumida na irrigação:

- Custo da energia $=\mathrm{R} \$ 0,17 \mathrm{kWh}^{-1}$

- Consumo específico médio $=173 \mathrm{kWh}\left(1000 \mathrm{~m}^{3}\right)^{-1}$

\section{RESULTADOS E DISCUSSÃO}

\section{Desempenho do modelo MCID - Esquema 1}

Os resultados das simulações conduzidas com o DRAINMOD e os da aplicação das metodologias de Pizarro e Cruciani, foram obtidos de Duarte (1997).

Os espaçamentos entre drenos laterais, obtidos com os modelos MCID e DRAINMOD e com as metodologias tradicionais de Pizarro (1985) e Cruciani (1987), são apresentados na Tabela 7. Obtiveram-se os espaçamentos indicados pelo DRAINMOD e pelo MCID com base em valores médios do valor presente líquido, para os 21 anos de simulação.

Os espaçamentos obtidos com o MCID foram maiores que os encontrados com o DRAINMOD, exceto para o Solo 3, para o qual os espaçamentos foram idênticos. Essas diferenças
Tabela 7. Espaçamentos entre drenos laterais $(\mathrm{m})$ obtidos com o MCID, DRAINMOD (DRA) e com as metodologias tradicionais de Pizarro (PIZ) e Cruciani (CRU)

\begin{tabular}{lcccc}
\hline \multicolumn{1}{c}{ Solo } & MCID & DRA & PIZ & CRU \\
Solo 1 & 50 & 45 & 16 & 22 \\
Solo 2 & 30 & 25 & 13 & 16 \\
Solo 3 & ${ }^{*} 10$ & $* 10$ & 5 & 4 \\
\hline Valor presente Irquido negativo & & &
\end{tabular}

ocorreram em virtude do déficit hídrico ter acarretado impacto consideravelmente maior sobre a estimativa da produtividade no MCID, em comparação com o DRAINMOD. Em simulações com o MCID e não se considerando o estresse devido ao déficit hídrico, os resultados para espaçamento entre drenos foram idênticos àqueles obtidos com o modelo DRAINMOD, apresentados na Tabela 7.

Em comparação com os espaçamentos obtidos por meio das metodologias de Pizarro e Cruciani, aqueles obtidos com o MCID e o DRAINMOD foram consideravelmente maiores; mais que o dobro para o Solo 1 e aproximadamente o dobro para os Solos 2 e 3, conforme a Tabela 7. Esta discrepância ocorre em razão dos critérios tradicionais de dimensionamento não considerarem o efeito da evapotranspiração sobre o rebaixamento do lençol freático, o estresse devido ao déficit hídrico, bem como da não observância do aspecto econômico nas metodologias tradicionais desconsiderando-se, assim, o fato de que o máximo agronômico não coincide, de modo geral, com o máximo financeiro. Também não é possível, utilizando-se apenas as metodologias tradicionais, verificar a viabilidade financeira do empreendimento (conforme indicado junto à Tabela 7 , para o Solo 3, o valor presente líquido negativo indica inviabilidade financeira).

As estimativas da produtividade relativa total da cultura, YRT (decimal), em termos das médias para os 21 anos de simulação, obtidas com o MCID e com o DRAINMOD, para os 
A.

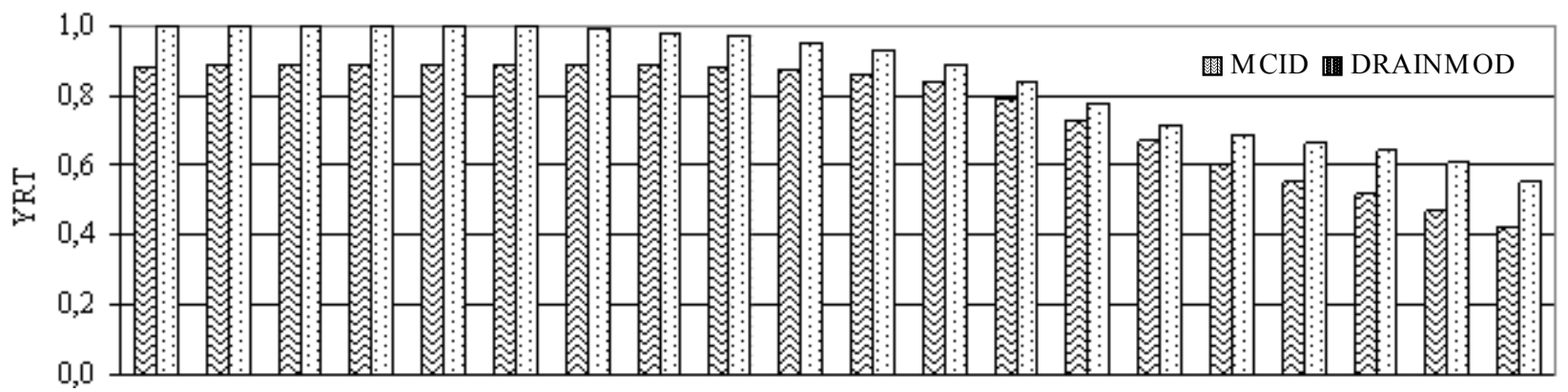

B.

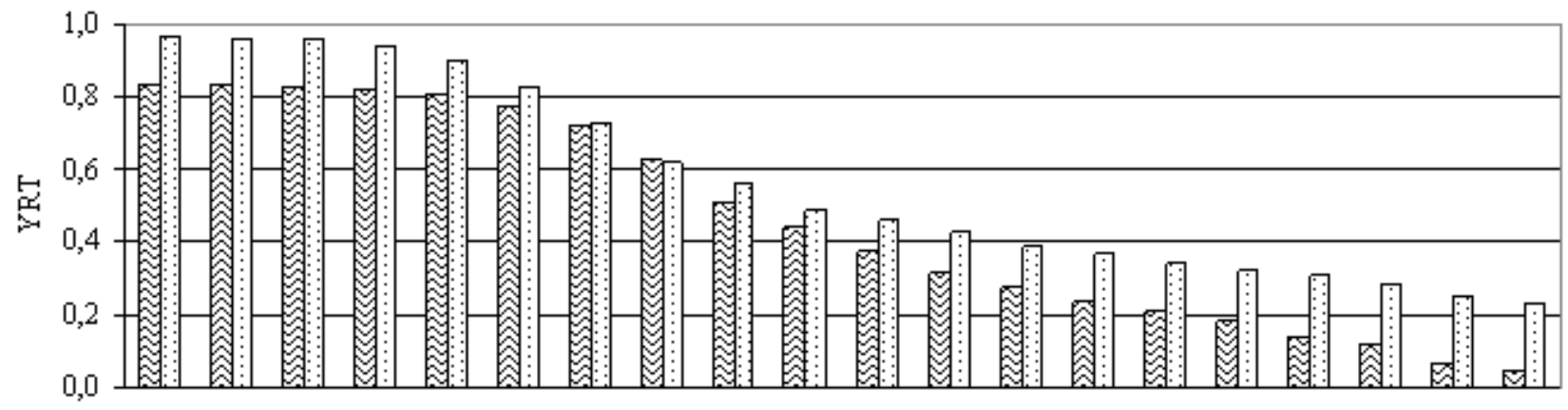

C.

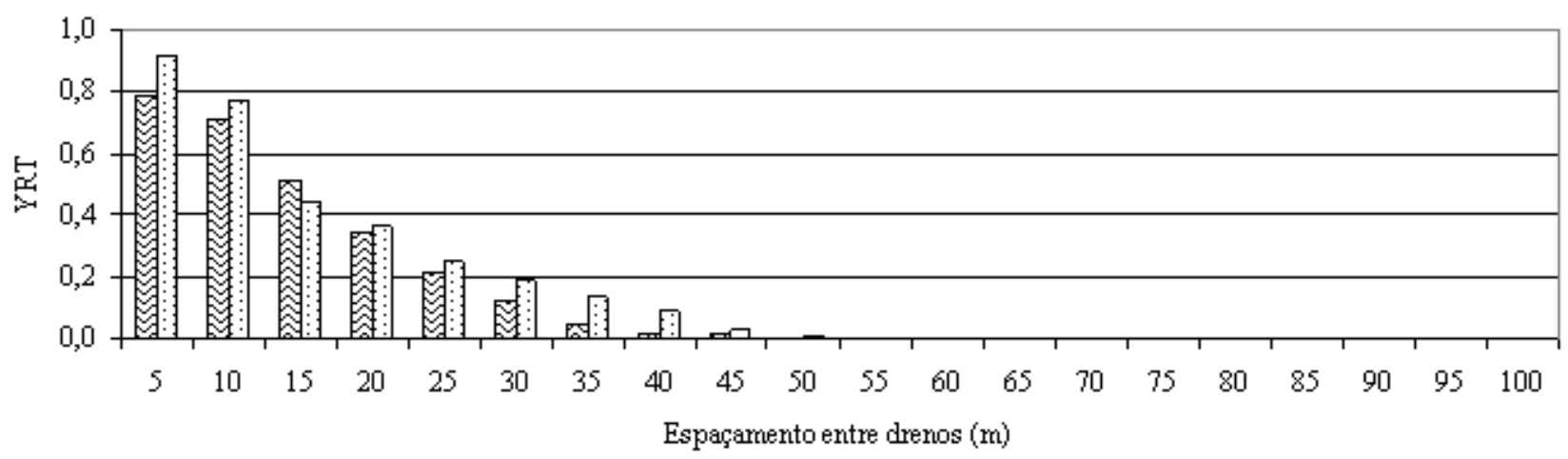

Figura 1. Produtividade relativa total da cultura (YRT, decimal) obtida com o MCID e o DRAINMOD, para os solos franco argiloso (A), argiloso (B) e franco argiloso-siltoso(C)

três tipos de solo, são apresentadas na Figura 1.

Em geral, YRT obtida com o MCID foi inferior à obtida com o DRAINMOD. Tomando-se como exemplo a Figura 1A, na qual são apresentados os resultados para o solo 1, observase que o DRAINMOD não previu qualquer decréscimo de produtividade até o espaçamento de $30 \mathrm{~m}(\mathrm{YRT}=1)$; isto indica que, para os menores espaçamentos entre drenos, para os quais não se verificou, com os dois modelos, estresse devido ao excesso de água na zona radicular, também não se detectou, com o DRAINMOD, estresse devido ao déficit hídrico, ou seja, a produtividade relativa em resposta ao déficit hídrico, (YRD), foi igual a 1. Por outro lado, resultados obtidos com o MCID indicaram que YRT e YRD, para espaçamentos até $30 \mathrm{~m}$, ficaram em torno de 0,89 .

As diferenças quanto aos procedimentos para estimativa da ETr, implementados no DRAINMOD e no MCID constituíram, provavelmente, a principal causa da diferença na estimativa de YRD e, por conseguinte, na estimativa de YRT (Figura 1). Segundo Skaggs (1981), no modelo DRAINMOD considera-se que a parcela da evapotranspiração real oriunda da zona radicular não depende do teor de água do solo ocorrendo, em sua potencialidade, até que o teor atinja o ponto de murcha permanente quando, então, passa a ser zero. No MCID considera-se um coeficiente de estresse devido ao déficit hídrico, $\mathrm{K}_{\mathrm{sd}}$ (Parte I deste artigo), o qual acarreta redução na taxa de evapotranspiração, conforme o decréscimo do teor de água do solo na zona radicular. Outra possível causa das diferenças nas estimativas de YRD é a não equivalência dos parâmetros relacionados à susceptibilidade da cultura ao déficit hídrico, utilizados como dados de entrada para os dois modelos.

\section{Desempenho do modelo MCID - Esquema 2}

Obtiveram-se, com os dois modelos, resultados muito próximos para produtividade relativa total (YRT) e evapotranspiração real acumulada para o ciclo (ETr). Para YRT foram obtidos os valores de 77,2 e 77,9\% e, para ETr, os valores de 253,2 e 253,3 mm, respectivamente, com o MCID e o CROPWAT.

Na Figura 2 tem-se os resultados de evapotranspiração real diária e, na Figura 3, os resultados de depleção de água na zona radicular e irrigações (intervalos e lâminas de irrigação foram dados de entrada). 


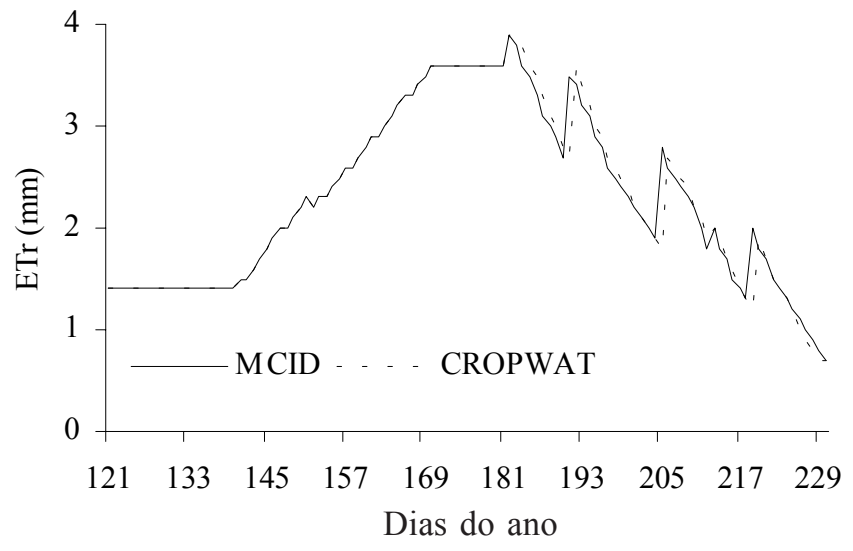

Figura 2. Evapotranspiração real obtida com o MCID e o CROPWAT

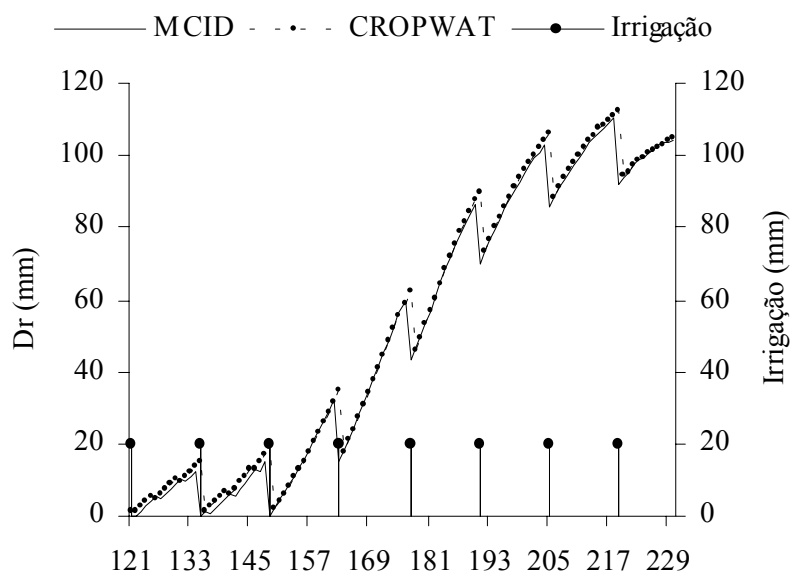

Dias do ano

Figura 3. Depleção de água na zona radicular (Dr) obtida com o MCID e o CROPWAT, e lâminas de irrigação

Nota-se na Figura 2, a partir do dia 181, uma defasagem entre os valores de ETr calculados com o MCID e o CROPWAT, devida ao modo como se processa a irrigação, nos dois modelos. No CROPWAT, a irrigação é computada no balanço hídrico no dia posterior à sua aplicação, o que é uma aproximação adequada quando a irrigação ocorre mais ao final do dia. No MCID, em virtude do procedimento iterativo implementado, a irrigação é processada no balanço hídrico no dia da aplicação. A defasagem entre os gráficos de ETr, obtidos com o MCID e o CROPWAT, não é observada até o dia 181 visto que, até então, a umidade do solo foi suficiente para sustentar a ETr igual à ETp, ou seja, a depleção de água na zona radicular era inferior à capacidade real de água no solo (CRA).

A diferença no processamento da irrigação refletiu, também, nos resultados para depleção de água na zona radicular, (Dr), apresentados na Figura 3, em que se observa defasagem nos dias de irrigação; na mesma figura, até o dia 149 (terceira irrigação), se observa que os valores de Dr, gerados pelo MCID, foram ligeiramente inferiores àqueles gerados pelo CROPWAT. Esta diferença é devida ao processamento da chuva (distribuição de valores mensais em base diária). No
CROPWAT, a primeira chuva do mês é alocada no primeiro dia do mês, enquanto no MCID é alocada no segundo dia; este fato, aliado à diferença de processamento da irrigação acima mencionado, acarretou ligeira diferença em Dr, apenas para o primeiro mês da simulação (mês 5), com o total de 12 mm de precipitação. A precipitação para os outros meses foi baixa (para os meses de junho a agosto, totalizou $4 \mathrm{~mm}$ ), não acarretando diferenças nas estimativas de Dr.

\section{Aplicação do MCID ao Projeto Jaíba}

Resultados obtidos com o MCID são apresentados na Tabela 8. O requerimento real de irrigação, (RI), nela apresentado, refere-se à parcela da irrigação que atinge a superfície solo, ou seja, corresponde à irrigação total menos as perdas por evaporação e arraste pelo vento. Nessas simulações, RI foi calculado com base, dentre outros parâmetros (Tabela 6), na eficiência de distribuição de projeto, (EDad), na qual está embutida a porcentagem da área que se pretende irrigar adequadamente e a uniformidade de aplicação (Keller \& Bliesner, 1990; Bernardo, 2005; Borges Júnior \& Mantovani, 2001).

Conforme se observa na Tabela 8 , os manejos de irrigação foram adequados quanto a prevenção ao déficit hídrico, sendo a ETr próxima ou igual à ETp e, conseqüentemente, os valores obtidos de YRT foram próximos ou iguais a 100\%. Para o RI são apresentados valores de média, desvio padrão e relativos aos níveis de 5 e $95 \%$ de probabilidade, sendo que o MCID fornece valores de RI e outras variáveis em diferentes níveis de probabilidade (Parte I do artigo).

Nesta tabela se verifica que alguns valores da relação $\mathrm{RI} / \mathrm{ETr}$ foram superiores a 1, como efeito da EDad considerada; por exemplo, para a cultura Feijão 2 (feijão no segundo ciclo - maio a julho), a EDad foi de 0,8, obtendo-se uma relação RI/ETr igual a 1,07.

Um exemplo de gráfico, gerado pelo MCID, com variáveis de saída relativas ao balanço hídrico, totalizadas para o ciclo

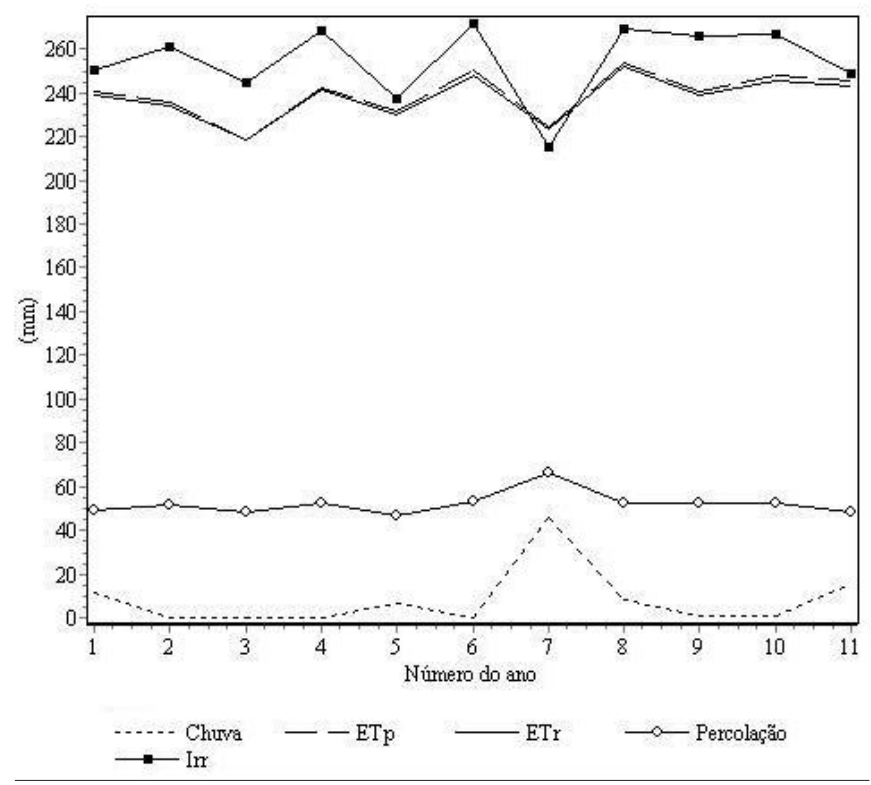

Figura 4. Gráficos gerados pelo MCID, de variáveis de saída relativas ao balanço hídrico, totalizadas para o ciclo da cultura Feijão 2: chuva, evapotranspiração potencial e real (ETp e ETr), percolação e irrigação real (Irr) 
Tabela 8. Valores acumulados de evapotranspiração real (ETr), evapotranspiração potencial (ETp), requerimento real de irrigação (RI - média, desvio padrão e valores equivalentes a 5 e $95 \%$ de probabilidade), requerimento total de irrigação (RIT), relação $\mathrm{RI} / \mathrm{ETr}$ e produtividade relativa total (YRT)

\begin{tabular}{|c|c|c|c|c|c|c|c|c|c|}
\hline \multirow[b]{2}{*}{ Cultura } & ETr & ETp & \multicolumn{4}{|c|}{ RI } & \multirow{2}{*}{$\begin{array}{c}\text { RIT } \\
(\mathrm{mm})\end{array}$} & \multirow[b]{2}{*}{$\mathrm{RI} / \mathrm{ETr}$} & \multirow{2}{*}{$\begin{array}{c}\text { YRT } \\
\text { média } \\
(\%)\end{array}$} \\
\hline & \multicolumn{2}{|c|}{$\begin{array}{c}\text { média } \\
(\mathrm{mm})\end{array}$} & média & desv. pad. & $p=5 \%$ & $p=95 \%$ & & & \\
\hline Banana ano 1 & 1.063 & 1.063 & 890 & 94 & 736 & 1.045 & 989 & 0,84 & 99,97 \\
\hline Banana ano 2 & 1.416 & 1.416 & 1.251 & 114 & 1.064 & 1.438 & 1.390 & 0,88 & 99,97 \\
\hline Mamão ano 1 & 958 & 958 & 791 & 46 & 715 & 867 & 791 & 0,83 & 100 \\
\hline Mamão ano 2 & 1.014 & 1.014 & 832 & 51 & 748 & 915 & 832 & 0,82 & 100 \\
\hline Mamão ano 3 & 497 & 497 & 395 & 30 & 345 & 444 & 395 & 0,8 & 100 \\
\hline Milho1 & 446 & 446 & 316 & 83 & 180 & 452 & 351 & 0,71 & 100 \\
\hline Milho2 & 332 & 332 & 397 & 22 & 356 & 434 & 442 & 1,2 & 100 \\
\hline Feijão 1 & 331 & 334 & 165 & 49 & 84 & 246 & 183 & 0,5 & 98,35 \\
\hline Feijão 2 & 238 & 239 & 255 & 18 & 226 & 284 & 283 & 1,07 & 98,43 \\
\hline Feijão 3 & 319 & 319 & 163 & 55 & 73 & 253 & 182 & 0,51 & 99,57 \\
\hline Cebola verão & 486 & 491 & 243 & 91 & 93 & 393 & 270 & 0,5 & 98,74 \\
\hline Cebola inverno & 370 & 375 & 403 & 43 & 333 & 474 & 448 & 1,09 & 98,67 \\
\hline Melancia & 327 & 327 & 305 & 30 & 255 & 354 & 305 & 0,93 & 100 \\
\hline Limão & 1.159 & 1.159 & 884 & 51 & 800 & 968 & 884 & 0,76 & 100 \\
\hline Pepino & 219 & 219 & 258 & 27 & 215 & 302 & 287 & 1,18 & 100 \\
\hline Algodão 1 & 313 & 313 & 288 & 53 & 200 & 376 & 320 & 0,92 & 100 \\
\hline Algodão 2 & 291 & 291 & 315 & 17 & 287 & 344 & 350 & 1,08 & 100 \\
\hline Abóbora japonesa & 304 & 304 & 204 & 65 & 97 & 310 & 226 & 0,67 & 100 \\
\hline Melão 1 & 273 & 273 & 256 & 24 & 216 & 296 & 256 & 0,94 & 100 \\
\hline Melão 2 & 315 & 315 & 303 & 27 & 259 & 348 & 303 & 0,96 & 100 \\
\hline Maracujá ano 1 & 314 & 314 & 245 & 22 & 209 & 281 & 245 & 0,78 & 100 \\
\hline Maracujá ano 2 & 869 & 869 & 660 & 38 & 598 & 722 & 660 & 0,76 & 100 \\
\hline
\end{tabular}

cultura Feijão 2, é apresentado na Figura 4, na qual se nota que a evapotranspiração real foi muito próxima à potencial, $\mathrm{o}$ que implicou em uma produtividade relativa média de 98,4\% (Tabela 8). Exceto para o ano 7, em que a precipitação foi pouco superior a $40 \mathrm{~mm}$, as lâminas percoladas variaram em torno de $50 \mathrm{~mm}$, condizentes com os valores obtidos de lâmina de irrigação real requerida, cuja média foi de $255 \mathrm{~mm}$ (Tabela 8), e com o valor considerado da eficiência de distribuição de projeto, (EDad), igual a 0,8 (Tabela 6), ou seja, a lâmina percolada deveria representar cerca de $20 \%$ da lâmina de irrigação real nos anos em que a chuva fosse inexpressiva durante o ciclo da cultura.

\section{CONCLUSÕES}

1. No teste comparativo com o modelo DRAINMOD, os espaçamentos entre drenos, obtidos com o MCID, foram maiores ou iguais aos obtidos com aquele modelo.

2. A produtividade relativa da cultura em resposta ao déficit hídrico obtida com o MCID foi, em geral, inferior àquela obtida com o DRAINMOD, acarretando menores valores da produtividade relativa total, YRT, nas estimativas com o MCID em relação aos obtidos com o DRAINMOD.

3. Em comparação com os espaçamentos obtidos por meio das metodologias de Pizarro e Cruciani, os obtidos com o
MCID e o DRAINMOD foram consideravelmente maiores.

4. Nos testes comparativos de produtividade relativa da cultura, evapotranspiração real (ETr) e depleção de água na zona radicular (Dr), entre o MCID e o programa CROPWAT da FAO, os resultados obtidos com os dois modelos estiveram muito próximos para YRT e ETr totalizadas para o ciclo da cultura.

5. Os resultados dos testes e da aplicação ao Projeto Jaíba indicam a potencialidade do MCID como ferramenta de apoio à tomada de decisão em projetos de irrigação e/ou drenagem.

\section{LITERATURA CITADA}

Allen, R.G.; Pereira, L.S.; Raes, D.; Smith, M. Crop evapotranspiration - Guidelines for computing crop water requirements. Rome: F.A.O., 1998. 300p. Irrigation and Drainage Paper 56.

Bernardo, S. Manual de irrigação. 7.ed. Viçosa: UFV, 2005. 611p. Borges Júnior, J.C.F. Modelo computacional para tomada de decisão em agricultura irrigada. Viçosa: UFV, 2004. 226p. Tese Doutorado

Borges Júnior, J.C.F.; Ferreira, P.A.; Pruski, F.F.; Silva Júnior, A.G. Sistemas de drenagem agrícola. Parte I: desenvolvimento do modelo e análise de sensibilidade. Revista Brasileira de Engenharia Agrícola e Ambiental, Campina Grande, v.5, n.3. p.369-375, 2001. 
Borges Júnior, J.C.F.; Hedden-Dunkhorst, B.; Ferreira, P.A. Decision support based on bio-economic simulations for irrigated agriculture. Deutscher Tropentag - Technological and institutional innovations for sustainable rural development. http://www.tropentag.de/2003/abstracts/full/224.pdf. 8-10, Out, 2003.

Borges Júnior, J.C.F.; Mantovani, E.C. Desenvolvimento de um programa para a avaliação da irrigação por sistemas pressurizados. In.: Mantovani, E.C.; Souza, L.O.C.; Soares, A.R. Engenharia na agricultura. Simpósio Brasileiro de Pesquisa em Cafeicultura Irrigada, 4, 2001, Araguari. Anais... Viçosa: UFV, 2001. p.42-48. Boletim Técnico $\mathrm{N}^{\circ} 4$

Cruciani, D.E. A drenagem na agricultura. 4.ed. São Paulo: Nobel, 1987. 337p.

Doorenbos, J.; Kassam, A.H. Yield response to water. Rome: FAO, 1979. 193p. Paper 33.

Duarte, S.N. Modelo para avaliação de desempenho de sistemas de drenagem subterrânea e cálculo de espaçamento de drenos. Viçosa: UFV, 1997. 143p. Tese Doutorado
Ferreira, P.A.; Borges Júnior, J.C.F.; Silva Júnior, A.G.; Pruski, F.F. Sistemas de drenagem agrícola. Parte II: Comparação entre o modelo desenvolvido e outras metodologias. Revista Brasileira de Engenharia Agrícola e Ambiental. Campina Grande, v.5, n.3. p.376-381, 2001.

Keller, J.; Bliesner, R.D. Sprinkle and trickle irrigation. New York: Avibook, 1990, 649p.

Pizarro, F. Drenaje agrícola y recuperación de suelos salinos. Madrid: Agrícola Española, 1985. 521p.

Quaresma Filho, M.I.M. Avaliação dos sistemas de irrigação por microaspersão na fruticultura dos projetos Gorutuba e Jaíba. Viçosa: UFV, 2000. 85p. Dissertação Mestrado

Skaggs, R.W. DRAINMOD - Reference report: Methods for design and evaluation of drainage-water management systems for soils with high water tables. Raleigh: USDA-SCS, 1981.329p.

Skaggs, R.W. Drainage simulation models. In: Skaggs, R.W.; van Schilfgaarde, J. (ed.) Agricultural drainage. Madison: Agronomy Society of America, 1999. cap. 13, p.469-500.

van Genuchten, M.T. A closed-form equation for predicting hydraulic conductivity of unsaturated soils. Soil Science Society America Journal, Madison, v.44, n.3, p.892-898, 1980. 